\title{
Efficient Remote Video Monitoring for Industrial Environment based on LPC2148 and GSM
}

\author{
T. Baghyasree ${ }^{1 *}$, K. Janakiraman ${ }^{2}$ and D. Parkavi ${ }^{1}$ \\ 'Embedded System, School of Computing, SASTRA University, Tirumalaisamudhram, Thanjavur-613 401, \\ Tamil Nadu, India; baghyasree@gmail.com, parkavi75@gmail.com \\ ${ }^{2}$ Shanmugha Polytechnic College, SASTRA University, Tirumalaisamudhram, \\ Thanjavur-613 401, Tamil Nadu, India; janakiraman@spt.sastra.edu
}

\begin{abstract}
Remote video monitoring system is one of the most important occasions for the entire industrial environment for security purpose through wireless networking. Wireless monitoring systems are used in many areas, especially for surveillance, monitoring and controlling the industrial operations. In day to day life, new technologies are arising for the purpose of industrial developing process in addition it needs the security system for maintenance. The main purpose of the proposed system is to protect the industries from risky situations. This system proposes a real time video monitoring device to detect the intruders which is based on ARMLPC2148 processor. Even so lots of video monitoring technology was available, but the integral system does not have the ability of perfect communication strategy. This work proposes an efficient system for monitoring the industrial environment by using a wireless camera to seize the video. When the ultrasonic sensor detecting the occurrence of the intruders or dynamic obstacle, instantly the Zigbee (CC2500) triggering the camera for video capture and GSM for sending SMS alerts to superior mobile for preventing the hazardous situation. The overall system performance is meliorating by using this technique and provides less power consumption, scalability, reliability and cost effective.
\end{abstract}

Keywords: CC2500, GSM, LPC2148, Mobile, Ultrasonic Sensor, Wireless Camera

\section{Introduction}

Now a day, human devote to their own safety, property protection and also pay attention in the working fields like to protect the plant equipment and worker safety. Ontogenies of economic system along with the enhancing of industries, security system are required to protect the industries from any damage as well as the human life. The Remote monitoring module plays a vital role in the security system. It deals with the protection process using wireless sensor network. In WSN (Wireless Sensor Network), the wireless communication systems have brought the visual sensation in real time. The wireless sensor network devices are humble in size and communicate in abruptly range. WSN will be increasing the development of low power, low cost and multi-functional sensors. Wireless network process is a major developing process for monitoring the industries ${ }^{14,16}$ without any distractions during maintaining of the environment. From remote locations, the physical environment will be proctored and control by battery powered nodes. From remote locations, the physical environment will be proctored and control by battery powered nodes. The WSN widely used and suitable ${ }^{9}$ for many real time applications such as agricultural, medical, Banking industry, military, industrial and environmental monitoring.

The basic security systems are gas leak alarm subsystem, fire alarm, anti-theft alarm system; monitor the temperature range, object detection. These are the usual security system, but it is used to intimate the alertness (i.e., Activate the buzzer) when the threshold value will beexceeded. The remote monitoring module's main purpose is indicating to which area was affected through capture the image using a camera. This process is used to prevent

*Author for correspondence 
the hazardous damage ${ }^{12}$ and also used as a immortalizeevidence of an offence for posterior inspection.

The remote monitoring system is mainly composed of alarm input, control unit and used to detect the object by using ultrasonic sensor. The sensors are used to monitor the environment conditions like temperature, pressure, light intensity, gas detector, and object detector. Remote proctoring and management is the major concern of the industrial environment for perfect controlling and satisfies the criteria of the industries. For every organization, a tolerant diversity of affordable video surveillance systems is usable. The video surveillance system device are depends upon the physical environment (an application). Video monitoring and smart storage solutions are embedded ${ }^{15}$ into the system. The choosing of components and the system functions depending on the proctor environment like camera counts, data retention, technology tolerance and budget, cost-effectiveness without compromising operation, the potentiality of the device.

The requirement of bandwidth is very high in video monitoring of industrial environment. This bandwidth requirement ${ }^{20}$ is the drawback of a remote monitoring system. The basic security system is the alarm message process and it depends upon the timestamp to send the message timely through GSM. With the eternal deployment of day to day life, the main attention is the human safety precaution during the monitoring process. However, the basic alarm system depends only the alerting ${ }^{11}$ message to the user which indicates the situation for human safety precaution, but it does not indicate which area was affected particularly.

In this remote video and image monitoring, easily find out which area was affected and also avoid from the uncontrollable situation for industrial environment. The embedded network ${ }^{10}$ technology deserves the environment monitoring process successfully. The embedded image and real time video monitoring system for industrial and surveillance and it is interfaced with the higher end ARM processor. This proposed system helps to protect the various environments like Industrial area, Home security, Banking industry, Wild animals and Agriculture and so on as shown in Figure 1.

\section{Related Work}

Wiliem et al. ${ }^{1}$ reported the process to detect the suspicious behaviour of humans. In video surveillance system, the CCTV camera is used to capture the video environment

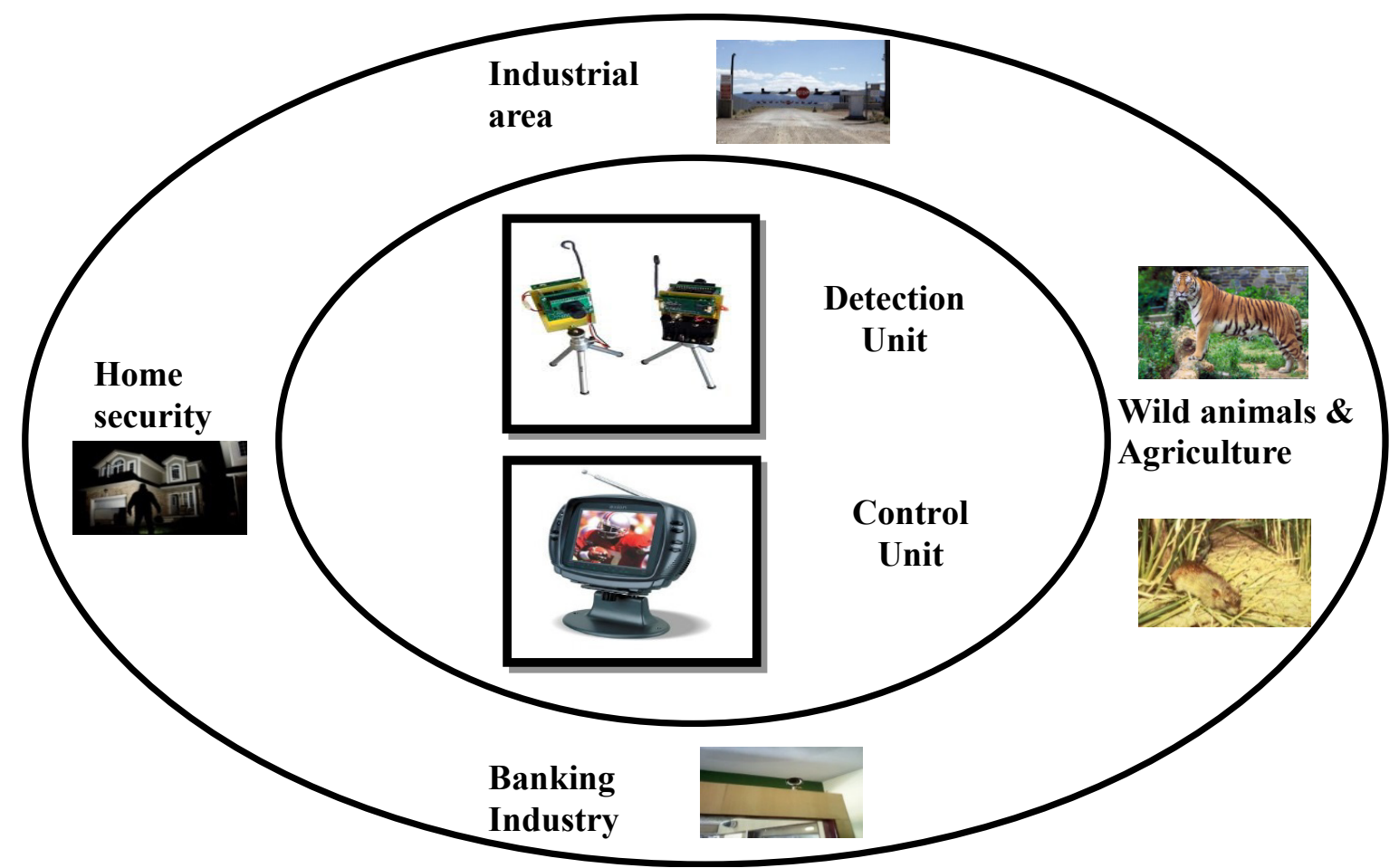

Figure 1. Various environments. 
to avoid lootings. Three main components are involved in this process: inference algorithm, context space model and data stream clustering algorithm. The comparative analysis performed between the previous context model and the current context space model. The videos are taken from the CAVIAR datasets and also it is used to compare the context space models. Contextual information segmented as two groups such as dependent groups and independent groups. The data stream clustering algorithm performs to retrieve the several patterns of human behaviour. The inference algorithm considers the current context information instead of previous context information. If the context space model was constructed, then the next process is to be identified the set representation of parameters. The set representation monitors the system context parameters, if any of the parameters alters then the context altering will be identified.

$\mathrm{In}^{2}$ method was developed a software authentication approach by LLE algorithm through Matlab. In this work, mainly focussed on the function of image identification for authentication purpose and calculate the eigen values and eigen vectors of the images. In this system, the predefined images are already stored into the database memory. The real time concurrent image will be captured and load into the system, which image has to be taken and calculate its eigen values and eigen vectors to compare with the predefined image of eigen values and eigen vector. The eigen value and eigen vectors depend on the expression of image, eye, nose, lips by using the LLE algorithm through Matlab software. The GUI (Graphical User Interface) was used to upload the concurrent image into the system. If the concurrent image eigen values and vectors are matched with the predefined image means, it will display the matching image of concurrent image by using GUI through Matlab and the comparative ratio of the image will be shown through the graph.

$\mathrm{In}^{3}$ methods were presented to monitor and control the industrial environment by using $\mathrm{ARM}^{19}$ processor S3C2410A and GPRS. The image acquisition process performs two steps. First, the picture captured through the USB camera and second, the enchanted pictures are collected through V4L, which is compressed by image compression algorithm. Because the BMP and PPM invades vast memory space and it is undesirable to transmit the vast picture $^{22}$ through internet. The picture compressed with JPEG compression. After that the compressed picture from a remote monitoring location transmits to local user through internet as well as the message indication will be sent to the user. The remote monitoring terminal is used to transmit the image, first collect the image and build the socket, i.e. the socket will be used to connect internet ${ }^{17,18}$ and transmit the compressed image. In compressed image, the identification of the object is difficult.

$\mathrm{In}^{4}$, ARM S3C2440 processor was built to control the overall process. In video monitoring system, the CMOS camera is involved in seizure the video continuously and here RGB565 is the format of the capturing video. The video pixel size is sixteen bits. The last five bits represent as blue element, the midsection six bits represent as green element and the upper section five bits represent as red element. In video capture module, the camera driver is an approach by "DeviceIoControl" in API. The video processing module main target is to detect the moving object from the video. When semi-permanent monitoring occurred, it reduces the affecting result from the exigency and the gradient in closed and dark light. The detected moving video is transmitted to the user mobile via email by video transmission module. The SMTP (Simple Mail Transfer Protocol) is used to the user phone receive the video through mailbox from monitoring area.

Tang et al. ${ }^{5}$ works process to remote monitor and control module based on ARM processor LPC2368 and GSM. The processor performs the function of monitoring the environmental conditions such as temperature range, humidity value and detects the harmful gas. Here, magnetic switch was built and the user enters the password through the keyboard. The authenticated person entered the correct password and it will be disabled the alarm system from the magnetic switch. When the abnormal situation will be occurring, instantly capture the image ${ }^{21}$ and sends to the user through MultiMedia Message (MMS). The controller sends the command to operate the GSM as AT command. GSM is used to store the image to the SD card through the command as ATUPLOADFILE and also send acknowledge.

$\operatorname{In}^{6}$ method was presented to proctor the human composite behavior by using state machines which try to avoid the robbery and effective support for a surveillance system. This system mainly focused on to detect the gesture based image feature like strange expression or exclaim expression and the image features linked to the state machine method. For identifying the object, two classes for motionless object and four classes for moving object. To determine the moving object, the system tracks the speed of the object and the trajectory of the object ${ }^{13}$ (direction). Here some patterns are loaded into the 
system to detect the various expressions of moving objects. When the strange behavior was detected, the alarm will be indicates and it depends upon the threshold level of patterns.

In ${ }^{7}$ method was developed to monitor and detect the stimulating object from the industrial environment by using Ubiquitous Sensor Networks (UBS). In this architecture, CCTV (Closed Circuit Television Camera) sensor node, RTU (Remote Telemetry Units) and a MS (the Management Server) are involved. The bi-directional data transferred between the sensor nodes and the MS by RTU. In WSN application, versatile sensor nodes are embedded in the industrial environment. The CCTV camera is used to capture the video and displayed on the PC.

$\mathrm{In}^{8}$ studies was mainly focused on capturing of image and the video by using CMOS (Complementary Metal Oxide Semiconductor). In this existing work, detect the motion object and capture the image of an object through the remote monitoring unit, which captured images of object sends to the mobile via Global System for Mobile Communications (GSM) network. The images and videos of motion object are compressed using image processing through hardware and transmit to the remote monitoring area for the surveillance system purpose. For detecting process, the motion detection algorithm is used and compares the video with the predefined image or background of the image. The comparison based on the pixel by pixel of current images and predefined image. This system could not provide the accurate detection process because it depends upon the algorithm based function.

\section{System Description}

Essentially the real time video monitoring is complex to implement in authoritative areas. The proposed system monitoring the crucial areas like industry, village, hospitals etc. It consists of two main modules: a) Detection Unit and b) Control Unit as shown in Figure 2. The Detection unit mainly focused on detecting the moving object, where objects enter into the restricted area. Detection unit and Control unit contains many modules as depicted in Figure 2. The Ultrasonic sensor, AT89S51 controller, Zigbee (CC2500), Relay switching and RF camera are embedded in the detection unit. As well as in the control unit, the Zigbee transceiver, LPC2148 processor, Buzzer, Relay switching, GSM and LCD are embedded. The LPC2148 ARM is a higher end processor to control the function of GSM process in the control unit.

\subsection{Detection Unit}

The ultrasonic sensor is interfaced with the AT89S51 controller and it is used to detect any kind of motion object within the specific distances. The specification of the ultrasonic sensor is to detect the motion object without any physical allusion by the target and is very sensitive. The ultrasonic sensor observes the object through the microcontroller and the intimation of object detection is transmitted to the control unit by using Zigbee TX/RX. As well the CC2500 trigger the relay switch to switch on the RF camera. The RF camera captures the industrial environment immediately.

\subsection{Control Unit}

The RF camera startles to seize the video of motion object, where the object crossing the limited surroundings in the restricted area. At the same time, the CC2500 transceiver of control unit received the signal from the detection unit and the control unit of CC2500 triggers the GSM for sending the alert message to the supervisor mobile, which does not make scathe for proles. Even the orator will be alarmed in the control unit for alert the proles and forbid them from scathe. The status of the system function will be continuously report in the LCD display and the captured video is transmitted to the control unit through an RF camera antenna. The RF tuner receives the transmitted video from the detection unit and the real time video is displayed on the television. The intimation of the moving objects detection is sent to the supervisor mobile through GSM, instantly. Supervisor can easily alert for security and also avoid the hazardous situations.

The design implementation of the overall system described through flowchart as depicted in Figure 3. The system utilizes the AT89S51 controller to initialize the ultrasonic sensor and trigger the camera at the presence of obstacles in the industrial environment. For the initialization of GSM module the LPC2148 processor is used to send the alert message.

\section{System Functioning and Evaluation}

Generally, all types of industries need security to maintain the system performance perfectly without any tragedy. For this security purpose various systems are developed, where the existing system does not have the ability to protect the industrial environment circumstances in addition 


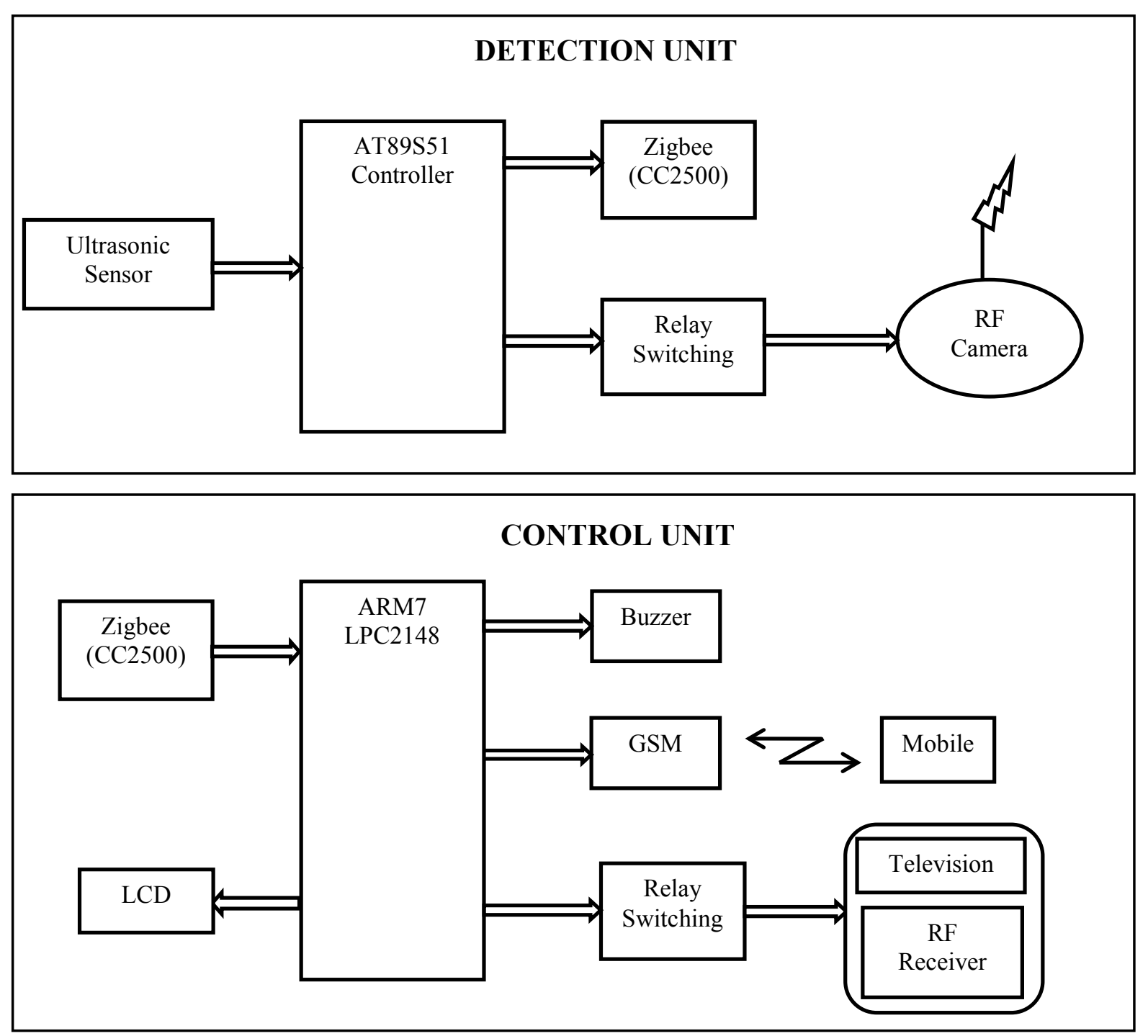

RESTRICTED AREA

Figure 2. System module.

to that, many drawbacks are occurring, namely less accuracy, high cost, difficult to design, no reliability and required more power consumption. In industrial environment, the major security alert system is the mobility detection to avoid the unnecessary calamity.

The proposed system provides two main units for remote monitoring, namely Detection unit and Control Unit. It overcomes the above drawbacks of existing systems. In detection unit, the Ultrasonic sensor is interfaced with the AT89S51 controller to detecting the mobility, presence in the restricted area of industries. The AT89S51 is a low power 8 bit microcontroller and high performance, which is very compatible with ultrasonic sensor for detection process. The ultrasonic sensor affords the high frequency sound pulses continuously at a standard time interval. When the pulses strike the object instantly it will generate the echo and the sensor received the echoes return by itself. By calculating the time interval between the transmitting and receiving signals, which is helping to detect the object occurrence and even also determine the distance of 
DETECTION UNIT

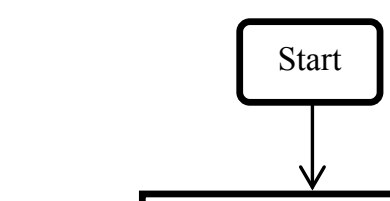

Initialize the

Ultrasonic Sensor

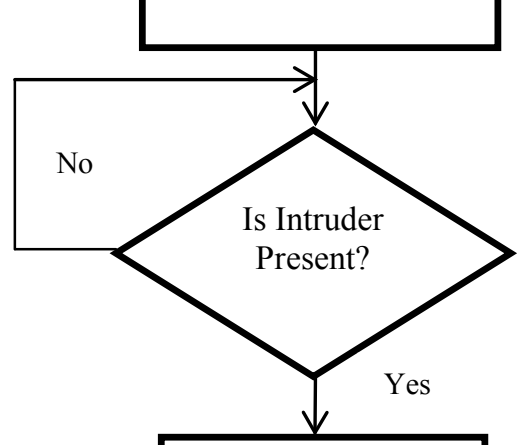

Sending the Signal

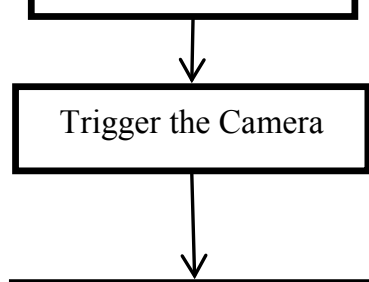

Sending the Real time video

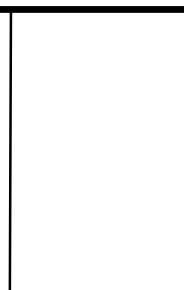

\section{CONTROL UNIT}

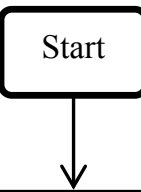

Initialize the GSM module

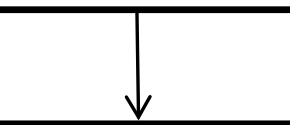

Waiting for Signal

Receive the Signal

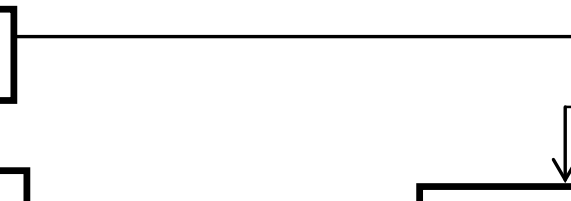

Report the Status in LCD

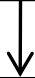

RF Receiver gets the video

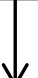

Display in TV

Stop

Figure 3. Function of system.

the object (within 10 meters). When the sensor detects the object, the Zigbee (CC2500) trigger the camera through a relay switch at the same time the buzzer will be alarmed and sends the signal for the purpose of alerting to the control unit. For saving the system's power, the camera will get ready to capture the video only after getting the signal from
Zigbee. Wireless Camera was admitted to performing a new technique and provides efficient real time remote video monitoring using Zigbee for industrial environment. The Zigbee is a low power - low cost, $2.4 \mathrm{GHz}$ RF device which is used for transmitting and receiving the signals and very compatible for industrial circumstances. 
The control unit main purpose is to communicate the system status of the industrial region to control room without collapse through GSM and LCD with the help of ARM7 LPC2148 higher end processor. The ARM7 is Advanced RISC Machine 32 bit microcontroller and low power consumption. Its operating voltage is $3.3 \mathrm{~V}$ and the operating frequency is up to $60 \mathrm{MHz}$ which contains two 10 bit ADC and one 10 bit DAC. ARM7 processor interfaced with LCD and GSM for sending SMS alerts to the superior of the control room in the industries. The control unit area of Zigbee receives the signal and triggers the GSM for alerting the superior through mobile via message. The status of the system function will be displayed on the LCD, even the absence and presence of obstacles also intimated by the help of LPC2148. To get a continuous monitoring of real time video, the RF tuner is used

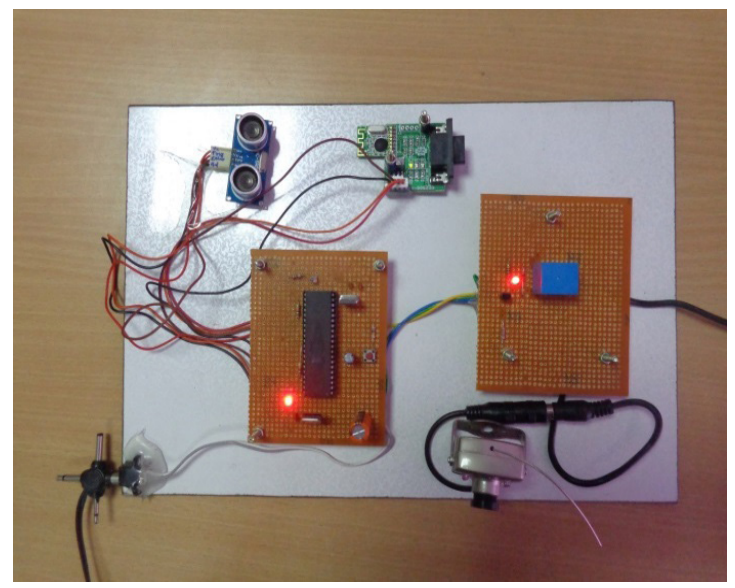

Figure 4. Detection unit.

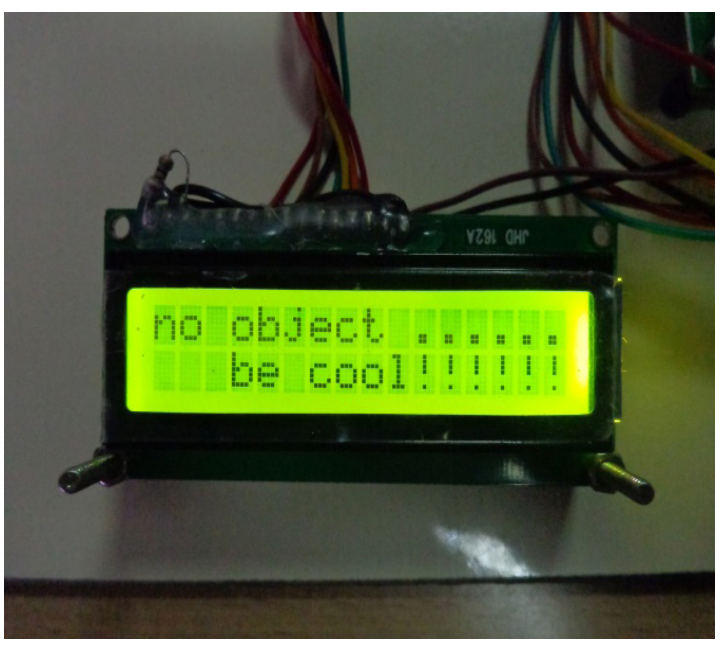

Figure 6. Absence of obstacles. to receive the video through $\mathrm{RF}$ antenna from the wireless camera in the detection unit. The captured real time video of environment will be ceaselessly monitored in the television by the superior. Using an RF antenna of video monitoring is better than the other transmitter because it is easily transmitted the video without any interrupt and even cost effective. The Effectuation takes place by a wireless camera and GSM through the combination of lower end and higher end controller. The hardware setup of Detection unit and control unit as depicted below in the Figure 4 and 5.

If no obstacle was presented in the industrial area then the status will be displayed as no object in the LCD as shown in Figure 6. When the object detected by the sensor, immediately the status of the system will be displayed as an intruder alert in the LCD as shown in Figure 7.

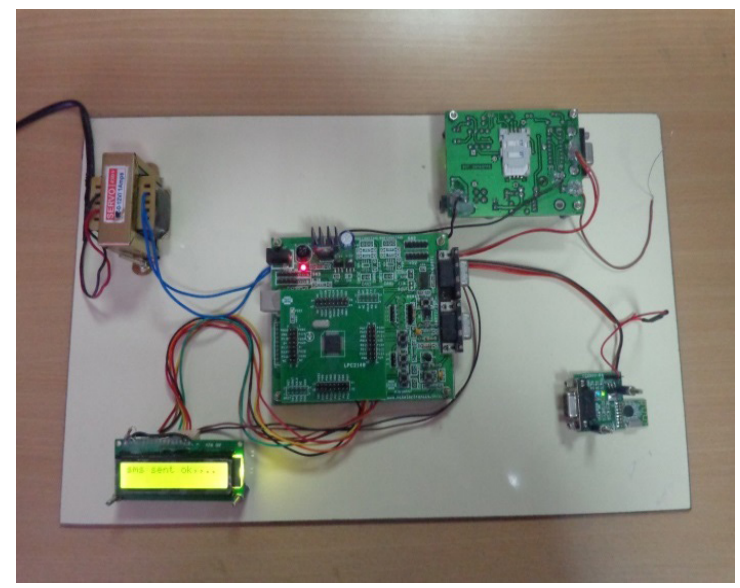

Figure 5. Control unit.

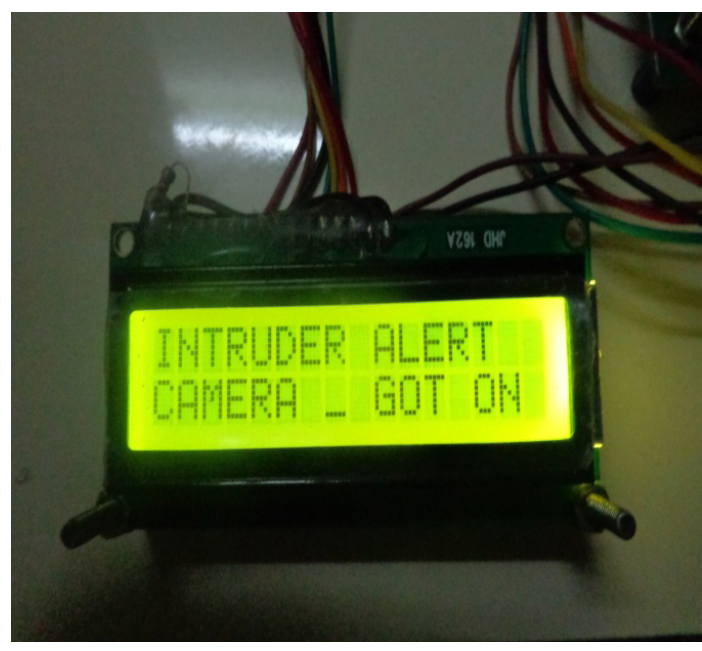

Figure 7. Presence of obstacles. 


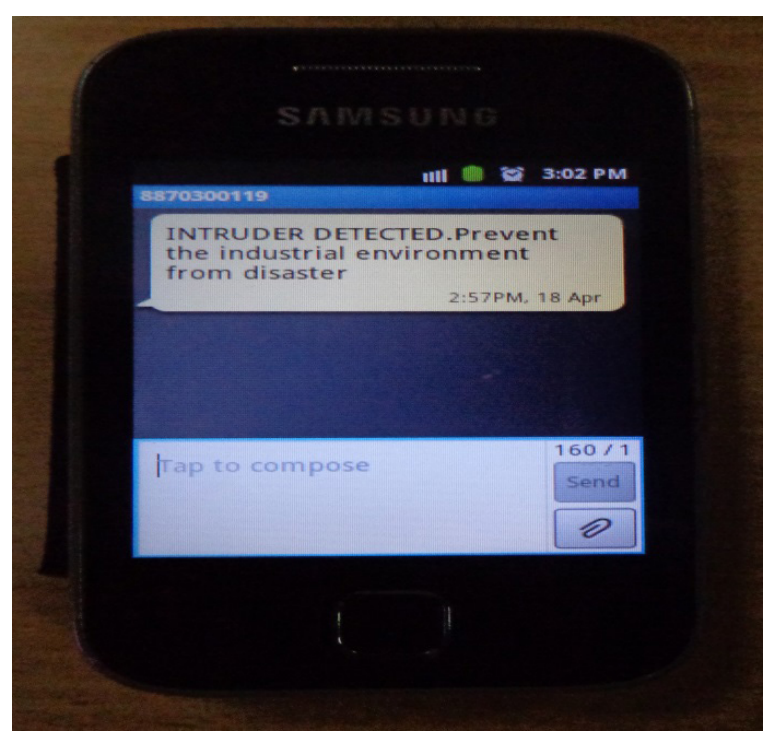

Figure 8. SMS Alerting through mobile.

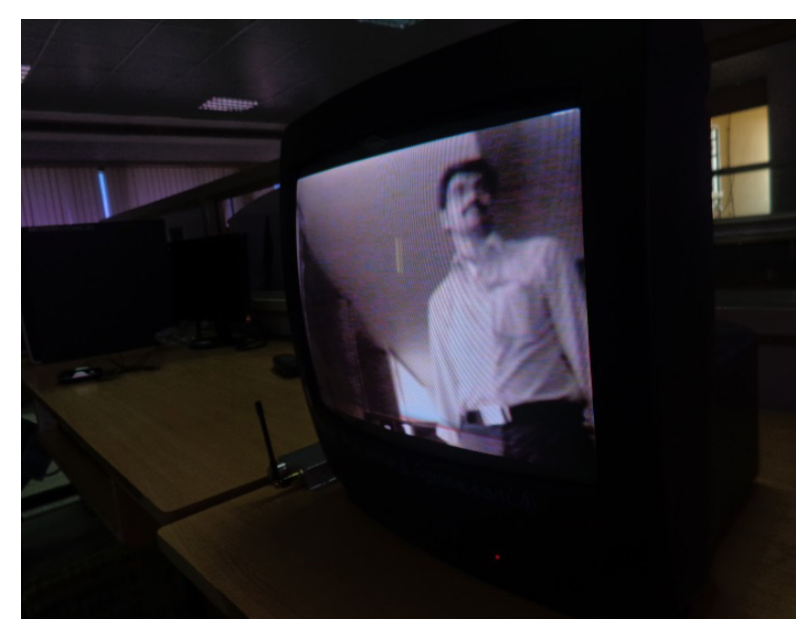

Figure 10. Video monitoring of darker area.

The object detected in the industries intimation will be sent as SMS alerting to the superior mobile through GSM from control unit is depicted in Figure 8. The wireless camera is embedded into the detection unit and captures the real time video. The real time video of industries environment will be displayed in the television by using the RF receiver as shown in Figure 9. The camera has the ability to seize the video even in the darker area and captured the real time video as shown in Figure 10.

\section{Conclusion}

Remote video monitoring process is the major concern for security purpose in industrial daily life. An expeditious

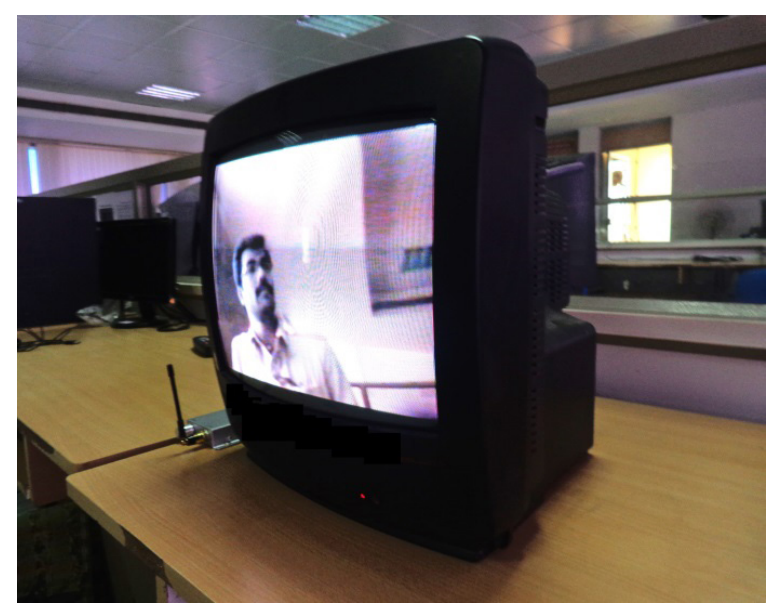

Figure 9. Video monitoring of environment.

remote monitoring system was developed for video surveillance of industrial environment by using LPC2148 and GSM for security purpose. This system proposes an untroubled real time video monitoring through the RF camera and provide better mobility detection within the distance of 10 meters. Comparison with other techniques, the detection unit communicates the control unit without fail and no data loss during the video capturing process. In the existing system, some trouble will be occurred on communication between systems. This new technology overcomes the existing technique problems and provides various advantages, namely less power consumption, better communication, accurate detection of obstacles, cost effective, compact in size and real time video monitoring. This proposed system has the capability to monitor the various diligences like industrial security, home security, banking industry, military, hospitals, and village and so on. In future, the system will be enhanced with the storage capacity of video monitoring for rectifying the fault occurring in the industries and easily find the intruder for further investigation.

\section{References}

1. Wiliem A, VamsiMadasu, Boles W, Yarlagadda P. A suspicious behaviour detection using a context space model for smart surveillance systems. Computer Vision and Image Understanding. 2012; 116:194-209.

2. Janakiraman K, Baghyasree T, Krithika N. Face Recognition for Authentication Purpose using LLE Algorithm. Int J Appl Eng Res. 2014; 9(2):203-12.

3. Fan D. The design and implementation of a wireless remote image monitoring system based on the ARM and GPRS. Procedia Engineering. 2011; 24:83-9. 
4. Chen H, Shen X, Mei F, YingdaLv. Embedded remote video surveillance system based on arm. CEAI. 2011; 13(3):51-7.

5. Tanga Z, Shuaib W, Luojun. Remote alarm monitor system based on GSM and ARM. Procedia Engineering. 2011; 15:65-9.

6. Fernández-Caballero A, Castillo JC, Rodríguez-Sánchez JM. Human activity monitoring by local and global finite state machines. Expert Syst Appl. 2012; 39:6982-93.

7. Song S. Design of emergency remote security monitoring and control system based on ARM. Consumer Electronics, Communications and Networks (CECNet); 2012 Apr 21-23; Yichang. IEEE; 2012. p. 2525-7.

8. Yu HY, Son SC, Kim NC, Oh SH, Ko SK, Park I, Yoon SK, Lee BT. Design and Implementation of a Video Surveillance System for a Sensor Network Application Service. 2012 Fourth International Conference on Ubiquitous and Future Networks (ICUFN); 2012 Jul 4-6; Phuket. IEEE; 2012. p. 494-7.

9. Marais J, Hancke GP. Design of a low cost video monitor store and forward device. Instrumentation and Measurement Technology Conference (I2MTC); 2012 May 13-16; Graz. IEEE; 2012. p. 165-70.

10. Zhao L, Huo C, Yang H. The design of remote video monitoring system based on S3C2416 and GPRS. 2012 International Conference on System Science and Engineering; 2012 Jun 30-Jul 2; Dalian, China. IEEE; 2012. p. 385-8.

11. Zhang X, Li X, Lu K. Remote video monitoring system based on ARM and Linux. 2012 Third International Conference on Intelligent Control and Information Processing; 2012 Jul 15-17; Dalian, China. IEEE; 2012. p. 369-72.

12. Rashmi R, Latha B. Video surveillance system and facility to access Pc from remote areas using smart phone. 2013 International Conference on Information Communication and Embedded Systems (ICICES); 2013 Feb 21-22; Chennai. IEEE; 2013. p. 491-5.

13. Benezeth Y, Laurent H, Emile B, Rosenberger C. Towards a sensor for detecting human presence and characterizing activity. Energ Build. 2011; 43:305-14.
14. FolgadoE, Rincon M, Carmona EJ, Bachiller M. A block-based model for monitoring of human activity. Neurocomputing. 2011; 74:1283-9.

15. Torabi A, Masse G, Bilodeau G-A. An iterative integrated framework for thermal-visible image registration, sensor fusion, and people tracking for video surveillance applications. Computer Vision and Image Understanding. 2012; 116:210-21.

16. Qi-An L, Peng L, Gui-zeng F, Ping L, Chang-lin L, Junxiao $\mathrm{Z}$. The solar-powered module design of wireless video monitoring system. Energy Procedia. 2012; 17:1416-24.

17. Ji G, Liu X, Zhang K. Design and implementation of remote model predictive control system. 2011 International Symposium on Innovations in Intelligent Systems and Applications (INISTA); 2011 Jun 15-18; Istanbul. IEEE; 2011. p. 19-23.

18. Al-Somaidai MB, Alsaydia OMA, Remote monitoring and controlling of gas sensors using VPN connection. 2012 International Conference on Future Communication Networks (ICFCN); 2012 Apr 2-5; Baghdad. IEEE; 2012. p. $84-7$.

19. Wang Z, Li W, Cao B, Jiang F. Design of the remote monitoring system for mine hoists. 2012 24th Chinese on Control and Decision Conference (CCDC); 2012 May 23-25; Taiyuan. IEEE; 2012. p. 3540-4.

20. Makwana R, Baviskar J, Panchal N, Karia D. Wireless based load control and power monitoring system. 2013 International Conference on_Energy Efficient Technologies for Sustainability (ICEETS); 2013 Apr 10-12; Nagercoil. IEEE; 2013. p. 1207-11.

21. Fang K, Tian Y. Touch Screen Monitoring System Based on CC-Link", 2013 International Conference on Computational and Information Sciences; 2013 Jun 21-23; Shiyang. IEEE; 2013. p. 1893-6.

22. Victor EL, Valenzuela, Vicente, Jazdi FN, Gohner P. Reusable hardware and software model for remote supervision of Industrial Automation Systems using Web technologies. 2013 IEEE 18th Conference on Emerging Technologies \& Factory Automation (ETFA); 2013 Sep 10-13; Cagliari. IEEE; 2013. p. 1-8. 